Bol. Inst. Oceanogr. Venezuela, Univ. Oriente 44 (2): 75-87 (2005); 7 Figs., 3 Tabs.

\title{
DISTRIBUCIÓN Y ESPECIACIÓN DE METALES PESADOS EN EL MATERIAL EN SUSPENSIÓN DE LAS AGUAS SUPERFICIALES DE LA PLUMA DEL RÍO MANZANARES, EDO. SUCRE, VENEZUELA
}

\author{
Gregorio Martínez, William Senior \& Arístide Márquez \\ Instituto Oceanográfico de Venezuela. Universidad de Oriente. Cumaná, Venezuela. \\ gmartine@sucre.udo.edu.ve
}

\begin{abstract}
RESUMEN: Se estudió la especiación de los metales pesados ( $\mathrm{Fe}, \mathrm{Mn}, \mathrm{Ni}, \mathrm{y} \mathrm{Pb}$ ) en el material en suspensión de las aguas superficiales de la cuenca baja y pluma del río Manzanares, Edo. Sucre, Venezuela, durante el período julio 1996 y junio 1997, en veintidós (22) estaciones establecidas en la cuenca baja y pluma del río. El material en suspensión se incrementó en los meses de lluvia (julio a noviembre), alcanzando el máximo valor en septiembre (1075 mg/l). Los flujos de metales pesados se incrementaron durante el período de lluvia, debido a su asociación con el material en suspensión. Las concentraciones totales de los metales en el material en suspensión de las aguas del río Manzanares fueron: Fe: 0,54-558,80 $\mu$ mol/1; Mn: 0,02-11,67 $\mu \mathrm{mol} / 1$; Ni: no detectado a $1,62 \mu \mathrm{mol} / 1$, y de no detectado a $0,17 \mu \mathrm{mol} / 1$ para el $\mathrm{Pb}$. En general, los metales evaluados presentaron un comportamiento no conservativo con remoción a bajas salinidades con desorción de los metales adsorbidos en la superficie de las partículas suspendidas y sedimentos, tanto orgánicas como inorgánicas, por intercambio con los iones $\mathrm{Na}^{+}$ y $\mathrm{K}^{+}$más abundante en el agua de mar, además de observarse aportes a todo lo largo de la pluma debido a las actividades humanas que se desarrollan en la zona. De igual manera, se determinaron concentraciones elevadas de $\mathrm{Mn}$ y $\mathrm{Pb}$ adsorbidos en el material en suspensión, como carbonatos y/o oxihidróxido de $\mathrm{Mn}$ reactivo, los cuales pueden ser incorporados más fácilmente a la cadena trófica o liberarse para ser incorporados de nuevo al agua, mientras que el Fe y $\mathrm{Ni}$ están mayormente asociados a los minerales de arcilla (principalmente aluminosilicatos), lo cual revela que el primer grupo de metales puede tener su origen en las actividades antrópicas que se desarrollan en la región que conforma la cuenca baja del río y su desembocadura.
\end{abstract}

Palabras clave: Metales pesados, especiación, río Manzanares.

Abstract: This paper reports on the speciation of $\mathrm{Fe}, \mathrm{Mn}, \mathrm{Ni}$, and $\mathrm{Pb}$ in the matter suspended in the superficial waters of the low basin and the surface trapped plume of the Manzanares river, in Cumaná, state of Sucre, Venezuela, by studying sediment and water samples taken at 22 stations deployed throughout this area of influence between July 1996 and June 1997. The suspended matter increased during the rainy season (July to November), reaching a maximum value in September, at 1075 $\mathrm{mg} / \mathrm{L}$. The heavy metal flow, in close association with the solid bodies in suspension, also increased during the rainy season, their total concentrations ranging from 0.54 to $558.80 \mathrm{imol} / \mathrm{L}$, from 0.02 to $11.67 \mathrm{imol} / \mathrm{L}$, from below detection to 1.62 ìmol/L, and from below detection to $0.17 \mathrm{imol} / \mathrm{L}$ for $\mathrm{Fe}, \mathrm{Mn}, \mathrm{Ni}$, and $\mathrm{Pb}$, respectively. Overall, the metals adsorbed to the surface of both organic and inorganic particles and sediments revealed a nonconservative behavior, evidencing wide removal at low salinities, namely, desorbing on account of the exchange with the most abundant ions in the sea wedge, $\mathrm{Na}^{+}$and $\mathrm{K}^{+}$. Human activity in the area kept a sustained and continuous contribution of materials to the river plume. Elevated concentrations of $\mathrm{Mn}$ and $\mathrm{Pb}$ are adsorbed to the suspended matter in the form of carbonates and/or reactive manganese oxide hydroxide, which allows for a less constrained incorporation to the trophic chain or freedom to return to the water mass, and is suggestive of their origin in the anthropic activities carried out in and around the watershed. The $\mathrm{Fe}$ and $\mathrm{Ni}$, on the other hand, are primarily associated to clay minerals mainly in the form of aluminosilicates.

Key words: Heavy metals, speciation, Manzanares river.

\section{INTRODUCCIÓN}

Los ríos son el principal vehículo de transporte de elementos químicos hacia el medio marino, especialmente de metales pesados. Numerosos estudios sobre el comportamiento de estos elementos químicos en estuarios, muestran que los procesos físicos, químicos, biológicos e hidrodinámicos que allí tienen lugar, cumplen un papel fundamental y variable en relación con el flujo de metales desde el continente hacia el mar (Zhang et al. 1992; YeATS,
1993). Igualmente, se ha reconocido que las descargas de los ríos influyen en la variabilidad espacial y temporal de los metales pesados (GibBs, 1977; Palanques \& Díaz, 1994). Por otra parte, los ríos contribuyen con aportes significativos de materia orgánica e inorgánica; los metales trazas que están asociados a la materia orgánica son liberados durante el proceso de degradación y lixiviación de los vertidos de materiales sólidos (MAGNusson \& Rasmussen, 1982; Valette-Silver,1993). 
En las últimas dos décadas se ha producido un gran cambio en nuestra comprensión de la biogeoquímica de los metales pesados en los ecosistemas marinos. La especiación de los metales gobierna las interacciones con la materia particulada en suspensión y los sedimentos, determina la biodisponibilidad y/o toxicidad de los metales para los organismos acuáticos, además de tener influencia en el ciclo de los metales a través de los estuarios, al igual que en la interfase, en ambientes con diferentes condiciones redox (LEWIS \& LANDING, 1992).

El río Manzanares constituye un cuerpo de agua de vital importancia para la región suroccidental del Estado Sucre y en especial para los centros poblados ubicados en sus márgenes, los cuales realizan diferentes actividades agropecuarias, industriales, de esparcimiento y, de igual manera, aprovechan sus aguas para las labores domésticas. En los últimos años estas actividades se han visto incrementadas considerablemente. Sus aguas reciben los efluentes de todas esas actividades sin ningún tratamiento y con poco control de las autoridades respectivas. Se ha prestado una mayor atención al comportamiento de los metales pesados en dicho río, donde se han reportado concentraciones totales de $\mathrm{Cd}$ y $\mathrm{Pb}$ moderadamente altas, de origen antrópico, que pueden afectar a los organismos de este ecosistema, y se ha establecido una relación directa entre la concentración de los metales pesados, el volumen de agua descargado por el río y su material en suspensión (LeÓn et al. 1997). Por otra parte, MÁrquez et al. (2000) estudiaron la distribución de algunos metales pesados en las aguas de este río, concluyendo que los metales en su mayoría se encuentran contenidos en el material en suspensión e igualmente asociados al gasto del río. De igual manera, Martínez \& Senior (2001) observaron, durante el período de lluvia, concentraciones elevadas de $\mathrm{Cd}, \mathrm{Cu}$ y $\mathrm{Zn}$ en el material en suspensión asociados a carbonatos y/u oxihidróxido de Mn mientras que el Cr está asociado principalmente a los minerales de arcilla (principalmente aluminosilicatos), lo cual puede indicar que el primer grupo de metales puede tener su origen en las actividades antrópicas que se desarrollan en la región que conforma la cuenca baja del río y su desembocadura. El presente estudio describe los procesos que gobiernan la distribución, especiación y comportamiento de los metales pesados ( $\mathrm{Fe}, \mathrm{Mn}, \mathrm{Ni}$ y $\mathrm{Pb}$ ) en el material en suspensión de las aguas superficiales de la cuenca baja y pluma del río Manzanares.

\section{AREAS DE ESTUDIO}

La cuenca del río Manzanares está ubicada en el Estado Sucre, Venezuela. Su vertiente está situada a 2.300 metros sobre el nivel del mar, en el macizo del Turimiquire y desemboca en la entrada del golfo de Cariaco donde ejerce gran influencia, sobre todo hacia el Oeste de la costa del golfo, cercana a la ciudad de Cumaná, entre los $10^{\circ} 24^{\prime}$ y $10^{\circ} 30^{\prime}$ Lat. N. y los $64^{\circ} 10^{\prime}$ y $64^{\circ} 20^{\prime}$ Long W. Su hoya hidrográfica cubre una extensión aproximada de $1.652 \mathrm{~km}^{2}$. $\mathrm{Su}$ aporte medio anual al mar es estimado en $558 \times 106 \mathrm{~m}^{3}$ de agua, la cual se reparte entre la zona del aliviadero y su desembocadura (Aguilera \& Rojas, 1976). El río Manzanares recibe las descargas de más de diez afluentes, donde el río Guasdua aporta el mayor número de contaminantes domésticos e industriales provenientes de los desechos de una central azucarera ubicada en la ciudad de Cumanacoa y los efluentes domésticos de los centros poblados de la región (Aguilera \& RojAs, 1976). Las descargas del río Manzanares dan origen a una pluma laminar cuyos límites forman un sistema semejante a un frente. La pluma se dirige, generalmente, hacia el Sur-Oeste como consecuencia de la predominancia de los vientos alisios. Ocasionalmente, cuando la descarga del río es mayor y los vientos alisios se intensifican, se deja sentir la influencia de la pluma en la región de Puerto Escondido en la costa al este de la bahía de Mochima ubicada a una distancia aproximada de $20 \mathrm{~km}$ desde la desembocadura (SEnior \& Godoy, 1989). El presente estudio se llevó a cabo en el último tramo de la cuenca baja y en la pluma del río (Fig. 1), las cuales se encuentran fuertemente afectadas por diferentes actividades antropogénicas.

\section{MATERIALES Y MÉTODOS}

Se recolectaron muestras de aguas superficiales, de manera bimensual, entre los meses de julio de 1996 y junio de 1997 en 22 estaciones, siguiendo el gradiente halino aproximadamente desde 0,0 en el puente Raúl Leoni, sector Las Palomas, hasta alcanzar salinidades superiores a 36,0 unidades en su pluma en dirección Sur-Norte. Las salinidades in situ fueron estimadas utilizando un salinómetro portátil YSI modelo 33 y luego fueron corregidas en el laboratorio utilizando un salinómetro de inducción marca Kahlsico, modelo 118WC200, con una precisión de 0,001 .

Las muestras de agua se filtraron con membranas 


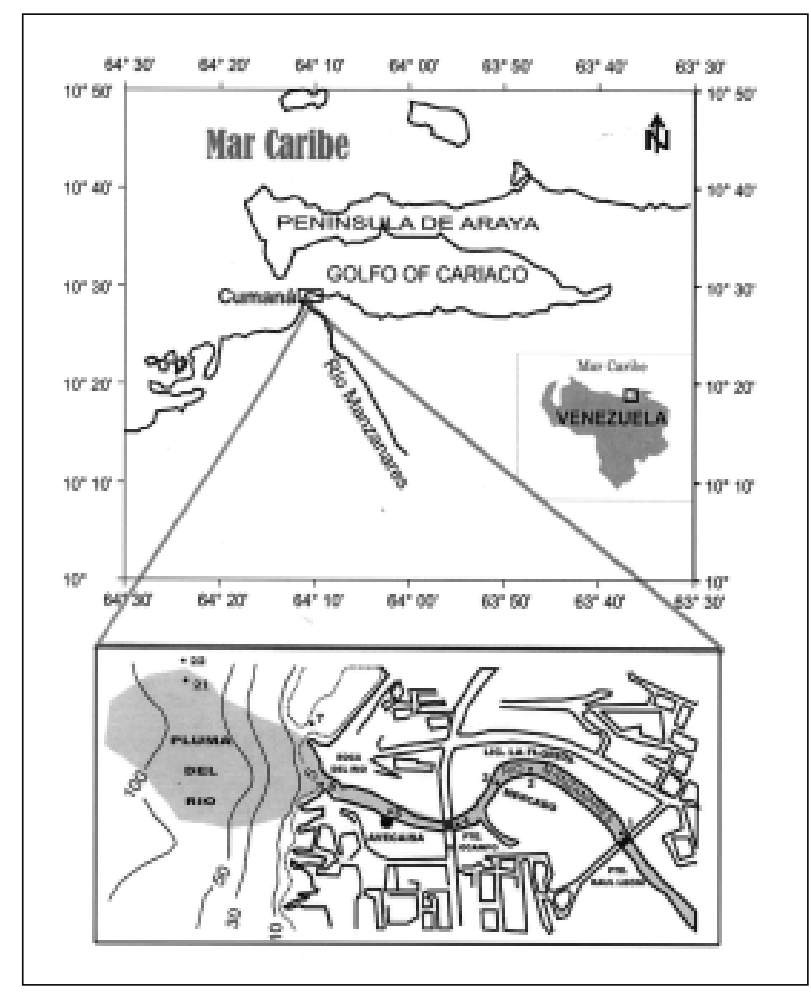

Fig. 1. Sector del río Manzanares y su desembocadura donde se llevó a cabo el estudio.

Millipore de nitrocelulosa con aberturas de poro $0,45 \mu \mathrm{m}$ previamente taradas, las membranas se secaron, repesaron y se sometieron a un procedimiento de extracción secuencial de tres pasos para la determinación de los metales pesados, de acuerdo a la metodología ideada por LANDING \& LeWIS (1991) y Lewis \& LANDing (1992), la cual consiste de una extracción con ácido acético al $25 \%$, por 4 horas a temperatura ambiente (fracción F1), seguido por una mezcla de "agua regia" diluida $\left(\mathrm{HCl} 2 \mathrm{~mol} / \mathrm{l}\right.$ y $\left.\mathrm{HNO}_{3} \mathrm{~mol} / \mathrm{l}\right)$ por 4 horas a temperatura ambiente (fracción F2). El material refractario que quedó en el filtro después de las extracciones se sometió a digestión a $100^{\circ} \mathrm{C}$ con una mezcla de los ácidos $\mathrm{HNO}_{3}, \mathrm{HCl}_{\text {y }} \mathrm{HClO}_{4}$, en una proporción 3:2:1, respectivamente (fracción F3).

La fracción F1 extrae los metales adsorbidos en la superficie de las partículas, asociados con los carbonatos y los oxihidróxidos de Mn reactivos, la fracción F2 extrae los oxihidróxidos de Fe más resistentes y sulfuros metálicos, mientras que la fracción F3 contiene los metales asociados a los minerales residuales refractarios y materia orgánica.
El material en suspensión (MES) se determinó de acuerdo a la metodología utilizada por Senior (1987). El contenido de metales en el MES se estimó a partir de la relación lineal $(\mathrm{y}=\mathrm{mx}+\mathrm{b})$ entre la concentración metal vs. el MES.

La precisión de este método fue evaluada utilizando una muestra estándar certificada de sedimento de la Environmental Resource Associates (Cat. No 1-800-3720122). Los valores de la desviación estándar y los coeficientes de variación son muy bajos, y además comparables entre las diferentes determinaciones, como se muestra en la Tabla I.

\section{RESULTADOS Y DISCUSIÓN}

Las representaciones gráficas de la relación entre los metales pesados y la salinidad muestran, para cada punto en la escala de salinidad, las concentraciones de los diferentes metales en cada una de las tres fracciones que

TABLA I. Precisión para el método de análisis de los metales pesados en el MES de las aguas superficiales del río Manzanares y su pluma. La concentración está expresada en mg/L.

\begin{tabular}{|c|c|c|c|c|}
\hline \multicolumn{5}{|c|}{ Ext.Ac. Acético $25 \%$} \\
\hline Muestra $\mathrm{N}^{\circ}$ & $\mathrm{Ni}$ & $\mathrm{Fe}$ & $\mathrm{Mn}$ & $\mathrm{Pb}$ \\
\hline 1 & 50,61 & 227,96 & 89,77 & 64,12 \\
\hline 2 & 50,17 & 228,67 & 90,32 & 64,75 \\
\hline 3 & 52,74 & 227,31 & 89,29 & 65,97 \\
\hline 4 & 51,64 & 226,59 & 89,44 & 65,66 \\
\hline 5 & 50,96 & 227,27 & 89,54 & 64,58 \\
\hline Promedio & 51,22 & 227,56 & 89,67 & 65,02 \\
\hline DS. & 1,0027 & 0,7883 & 0,4017 & 0,7740 \\
\hline \multicolumn{5}{|c|}{ 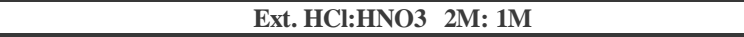 } \\
\hline Muestra $\mathrm{N}^{\circ}$ & $\mathrm{Ni}$ & $\mathrm{Fe}$ & $\mathrm{Mn}$ & $\mathrm{Pb}$ \\
\hline 1 & 9,34 & 359,69 & 12,08 & 0,00 \\
\hline 2 & 9,23 & 359,66 & 11,85 & 0,00 \\
\hline 3 & 9,30 & 360,12 & 12,03 & 0,00 \\
\hline 4 & 9,17 & 362,98 & 12,02 & 0,00 \\
\hline 5 & 9,19 & 360,75 & 11,67 & 0,00 \\
\hline Promedio & 9,25 & 360,64 & 11,93 & 0,00 \\
\hline DS. & 0,0722 & 1,3832 & 0,1662 & 0,0000 \\
\hline \multicolumn{5}{|c|}{ Ext. Residuo (Mezcla de HNQ:HCl:HClO 4 3:2:1) } \\
\hline Muestra $\mathrm{N}^{\mathrm{o}}$ & $\mathrm{Ni}$ & $\mathrm{Fe}$ & $\mathrm{Mn}$ & $\mathrm{Pb}$ \\
\hline 1 & 0,00 & 11746,86 & 58,78 & 20,76 \\
\hline 2 & 0,00 & 11709,90 & 60,49 & 21,50 \\
\hline 3 & 0,00 & 12108,55 & 65,31 & 19,13 \\
\hline 4 & 0,00 & 11896,79 & 61,68 & 18,62 \\
\hline 5 & 0,00 & 11849,63 & 59,59 & 19,85 \\
\hline Promedio & 0,00 & 11862,35 & 61,17 & 19,97 \\
\hline DS. & 0,0000 & 156,9576 & 2,5521 & 1,1749 \\
\hline
\end{tabular}


fueron extraídas del material en suspensión (MES) y la concentración total (suma de las áreas), dada por el contorno superior. Los cuatro primeros puntos corresponden a las estaciones ubicadas dentro del río Manzanares, el penúltimo al frente del río (interfase agua de río-mar) y el último al extremo marino (fuera del frente). Los puntos entre el extremo fluvial y el frente corresponden a la zona de mezcla.

\section{Hierro}

La Fig. 2, muestra la distribución del hierro contenido en el MES, en relación con la salinidad. Se presenta un comportamiento no conservativo con las mayores concentraciones en el extremo fluvial donde los aportes son altos en la zona mezcla del agua dulce con la marina en la pluma del río como consecuencia del aumento de la fuerza iónica y del $\mathrm{pH}$, precipitando el hierro y el manganeso como oxihidróxidos (formación de fase sólida). De igual manera, las concentraciones de Fe en el MES fueron mayores durante el período de lluvia como resultado del aumento en el gasto del río y el material en suspensión, alcanzando valores en el mes de septiembre de $6,92 \mu \mathrm{mol} /$ 1 los biodisponibles (F1), 59,6 $\mu \mathrm{mol} / 1$ para los oxihidróxidos de manganeso y sulfuros metálicos (F2) y 359,08 $\mu \mathrm{mol} / \mathrm{l}$ para los materiales refractarios (F2), en el extremo fluvial, mientras que en el extremo marino las concentraciones tienden a cero. La relación entre las concentraciones del Fe en el MES y el material en suspensión mostró una correlación positiva altamente significativa $(\mathrm{r}=0,975, \mathrm{p}<$ $0,05)$ durante todo el todo el período de lluvia (Tabla II), mientras que en el período de sequía no se observó correlación, posiblemente debido al dragado de la región donde desemboca el río. De igual manera, las concentraciones asociadas al material residual que corresponden al Fe combinado con los cristales de los minerales y materia orgánica que conforman las partículas de sedimentos se presentaron en una proporción mayor del $50 \%$ del total en todos los meses de muestreo, lo que evidencia el alto contenido de hierro en las arcillas y otros minerales presentes en corteza terrestre de la región que conforman la Hoya hidrográfica del río Manzanares.

El comportamiento de Fe en el MES está de igual manera asociado al pH, lo que sugiere que los procesos de adsorción, floculación o formación de coloides tienen influencia en la precipitación de Fe como oxihidróxido a $\mathrm{pH}$ mayor de 8, puesto que a valores menores dicho elemento permanece en solución (Fig. 3). El mismo

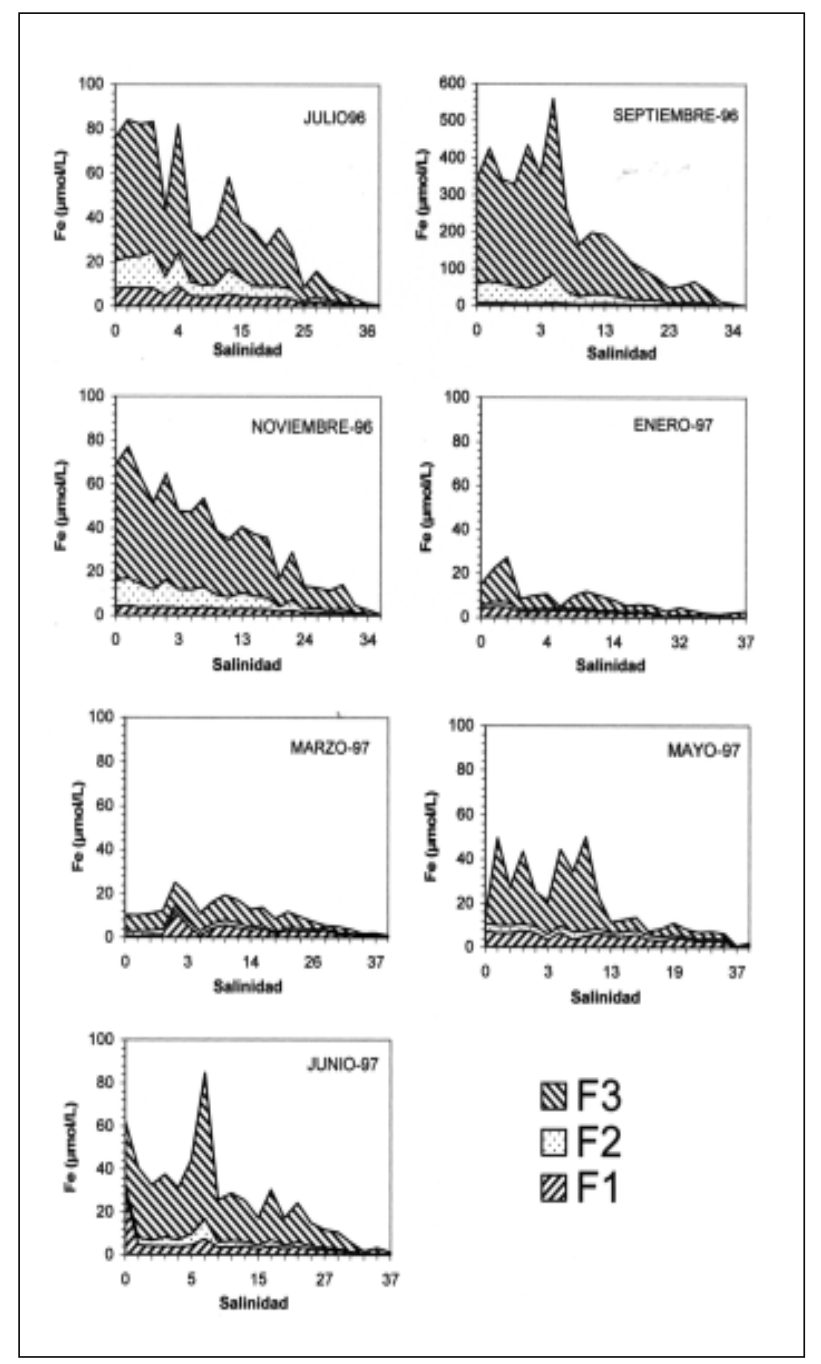

Fig. 2. Relación entre el Hierro en el MES y la salinidad en las aguas superficiales del río Manzanares y su pluma durante el muestreo.

TABLA II. Matriz de correlación de Pearson que muestra las correlaciones entre el material en suspensión, el pH y los metales pesados presente en las aguas superficiales de la cuenca baja y pluma del río Manzanares durante el mes de septiembre de 1996 (21 observaciones).

\begin{tabular}{lcccccc}
\hline & $\mathrm{pH}$ & $\mathrm{MES}$ & $\mathrm{Fe}$ & $\mathrm{Mn}$ & $\mathrm{Ni}$ & $\mathrm{Pb}$ \\
\hline $\mathrm{pH}$ & 1.000 & & & & & \\
MES & 0.742 & 1.000 & & & & \\
$\mathrm{Fe}$ & 0.730 & 0.975 & 1.000 & & & \\
$\mathrm{Mn}$ & 0.674 & 0.817 & 0.815 & 1.000 & & \\
$\mathrm{Ni}$ & 0.786 & 0.986 & 0.971 & 0.884 & 1.000 & \\
$\mathrm{~Pb}$ & 0.826 & 0.893 & 0.895 & 0.820 & 0.933 & 1.000 \\
\hline
\end{tabular}




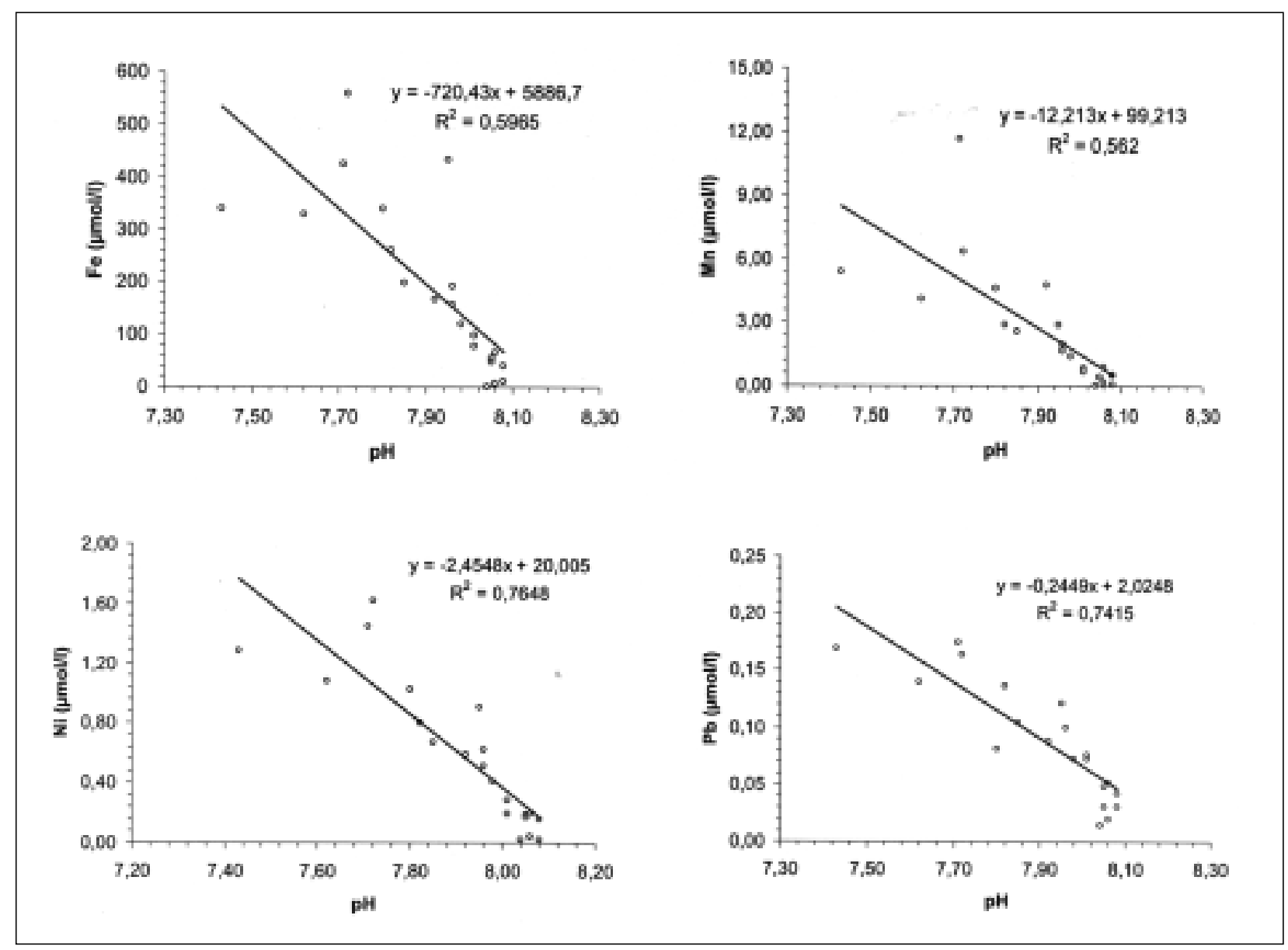

Fig. 3. Relación entre la concentraciones de los metales $\mathrm{Fe}, \mathrm{Mn}$, $\mathrm{Ni} \mathrm{y} \mathrm{Pb}$ con el pH en las aguas superficiales de la cuenca baja y pluma del río Manzanares durante el mes de septiembre de 1996.

comportamiento observaron MARTíNEZ \& SENIOR (2001) para los metales $\mathrm{Cd}, \mathrm{Zn}, \mathrm{Cu}$ y $\mathrm{Cr}$ en las aguas del río Manzanares y su pluma para el mismo período de tiempo. Este fenómeno sucede en los primeros momentos de la mezcla del agua del río con las marinas, debido a los cambios en la fuerza iónica y pH de la solución, ocurriendo la floculación de los coloides y precipitación de los oxihidróxidos de hierro y manganeso. En otros escenarios, por ejemplo, en los río Jiulugjiang y Changjian (China) el Fe en el MES es removido a salinidades menores a 20, atribuyéndose esto a la floculación coloidal, manteniéndose conservativo a altas salinidades (ZHANG, 1995).

Remociones entre 15 y $67 \%$ en el período de lluvia y 12 a $53 \%$ en sequía para el $\mathrm{Fe}$ en el MES fueron reportadas por MÁRQUEZ (1997) para el mismo río Manzanares, mientras que LEÓN (1995) y LEÓN et al. (1997) observaron remociones de Fe total menores de $40 \%$ durante el período de lluvia y entre 60 y $70 \%$ en la temporada de sequía. MARTIN \& Windom (1990) mostraron incrementos del hierro en los revestimientos de las partículas de arcillas en el estuario Gironde (Francia), atribuyendo ésto a la turbidez máxima en el estuario y a la asociación del metal con compuestos orgánicos y partículas de arcilla que floculan a salinidades entre 6 y 8. COONLEY et al. (1971) encontraron remoción de Fe a bajas salinidades en el estuario del río Mullica y Bahía Great (Nueva Jersey EEUU) debidos a cambios en el pH e incremento de la salinidad. HART Y DAVIS (1981) determinaron pérdidas de hierro de $50 \%$ a salinidades entre 0 y 2 en el río Yarrah (Australia) debido a que dicho elemento se encontraba ligado a complejos húmicos y al material en suspensión, produciéndose la floculación del mismo debido 
a pequeños cambios del pH y a la fuerza iónica. De igual manera, MARTIN et al. (1993) indicaron que la remoción del hierro a bajas salinidades ocurre por la floculación y precipitación de los oxihidróxidos coloidales debida a los cambios en el pH y la fuerza iónica. No obstante, las cantidades de este elemento siempre fueron mayores en el extremo fluvial, lo que indica que el río Manzanares se constituye, con su drenaje, en la principal fuente de este metal al ecosistema estudiado.

\section{Manganeso}

Al igual que para los demás metales estudiados, el manganeso contenido en el MES se presenta en la Fig. 4, con un comportamiento no conservativo, con las mayores concentraciones en el extremo fluvial con remoción a salinidades bajas y algunos aportes a lo largo de la pluma. Además, las variaciones estacionales en las concentraciones de Mn del MES están muy relacionadas con el gasto de río y el material en suspensión (Tabla II), observándose los mayores valores durante el mes de septiembre $(0,008-9,32 \mu \mathrm{mol} / 1$ para el $\mathrm{Mn}$ asociado a F1; $0,006-1,41 \mu \mathrm{mol} / 1$ para la F2; 0,003-0,94 $\mu \mathrm{mol} / 1$ en la F3 y los menores en el mes de enero $(0,09-0,144 \mu \mathrm{mol} / 1$ para el Mn en la F1 ; de no detectado a $0,027 \mu \mathrm{mol} / 1$ para F2; 0,017-0,068 $\mu \mathrm{mol} / 1$ en la F3). En los meses de julio y septiembre se observó remoción del Mn del MES con aportes en algunos puntos de la pluma, mientras que en el mes de noviembre la concavidad de la curva se invierte indicando adición de Mn desde el MES a la pluma, posiblemente debido a desorción de Mn de la superficie de las partículas en suspensión. En enero, las concentraciones de dicho metal variaron poco a lo largo de la pluma como consecuencia del dragado en la desembocadura del río. En marzo y mayo se observan aportes entre 0 y 6 de salinidad debido a la penetración de la cuña salina dentro del río aumentando la formación de oxihidróxidos de $\mathrm{Fe}$ y $\mathrm{Mn}$, donde se presentaronn fuertes vertidos de efluentes líquidos con un alto contenido de materia orgánica. En junio, se presentaron aportes en la zona de mezcla producto de la llegada de las primeras lluvias que lavan la corteza terrestre. Igualmente, se observó una mayor proporción de Mn biodisponible en el MES, superior al $60 \%$ durante todos los muestreos, mientras que las fracciones asociadas a los oxihidróxidos de $\mathrm{Fe}$ resistentes, sulfuros metálicos y materiales refractarios presentaron las menores proporciones. Esto puede sugerir que el manganeso está siendo incorporado al sistema a partir de los vertidos de desechos industriales y

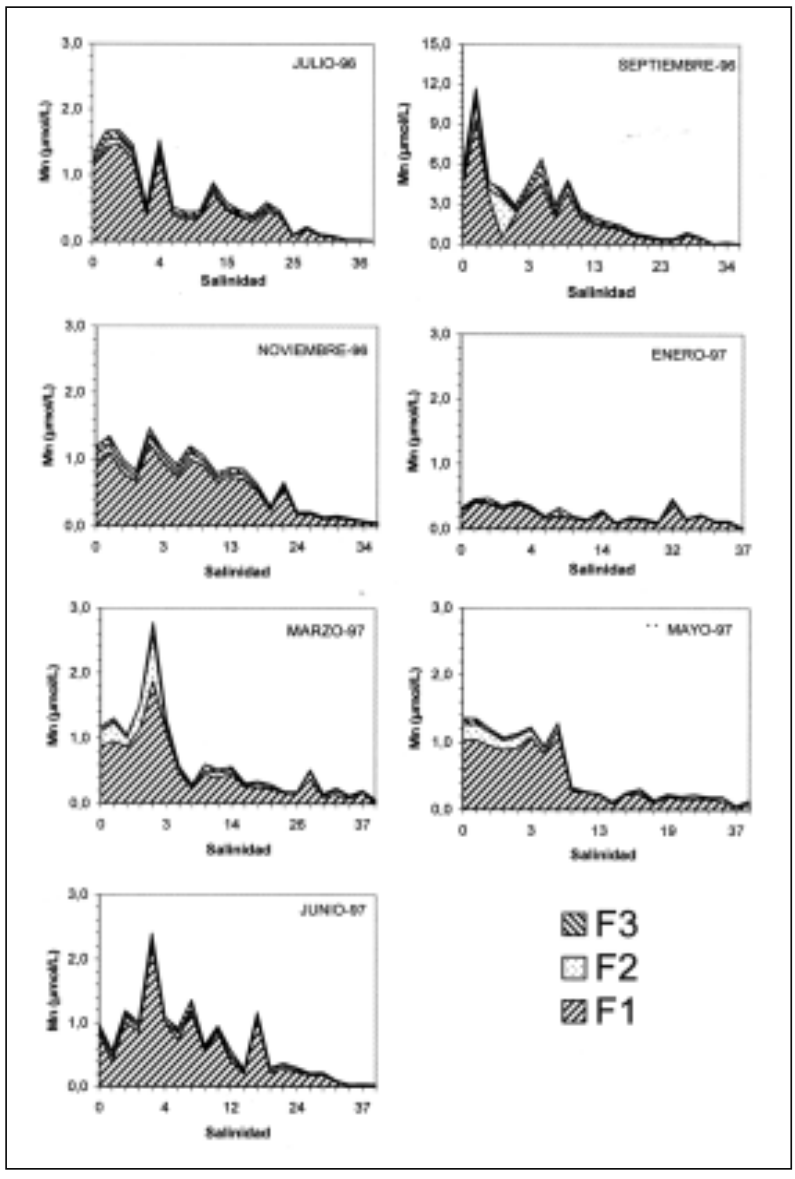

Fig. 4. Relación entre el Manganeso contenido en el MES y la salinidad en las aguas superficiales del río Manzanares y su pluma durante el presente estudio. Las magnitudes en la escala del eje y $(\mu \mathrm{mol} / \mathrm{l})$ cambian para el mes de septiembre.

domésticos, muy ricos en materia orgánica, con poco o ningún tratamiento, fertilizantes utilizados en las actividades agrícolas y demás actividades antropogénicas desarrolladas en las márgenes del río Manzanares. Por otra parte, el Mn al estar adsorbido y revistiendo las partículas de arcilla que conforman el material en suspensión puede liberarse fácilmente cuando comienzan a mezclárse las aguas dulces con las marinas, intercambiándose por iones sodio, muy abundantes en el agua de mar. Esto produce procesos de desorción, floculación, formación de oxihidróxido de manganeso y precipitación de Mn (formación de fase sólida) a lo largo de la pluma del río.

MÁrquez (1997) reportó un comportamiento no conservativo del Mn en el MES para este mismo río con remociones entre 26 y $55 \%$ en el período de lluvia y $14-67 \%$ 
en sequía con aportes que variaron entre 0,14 y $5,55 \mu \mathrm{mol} /$ 1, con una correlación muy significativa con el material en suspensión transportado por el río. Resultados similares fueron encontrados por LEÓN (1995), quien reportó alta correlación en los meses de agosto y noviembre. Otras observaciones similares presentaron CARPENTER et al. (1975) para la bahía de CHESAPEAKE (EEUU), y GibBs (1977) en el río Amazonas.

Mas aún, el comportamiento observado del manganeso del MES en este estudio es muy similar al del hierro del MES, principalmente en el período de mayor caudal del río, evidenciándose por la correlación muy significativa que existe entre estos dos elementos (Tabla II). Graham et al. (1976) indicaron que el comportamiento del Mn está influenciado por las condiciones fisicoquímicas que prevalecen en cada estación del año, mientras que MoRris \& BALE (1979), señalaron que la adsorción y coprecipitación de este elemento con los oxihidróxidos de hierro están asociadas a la disminución del pH, aumento de las concentraciones de oxígeno e incremento de la fuerza iónica. El mismo resultado fue reportado por LEón (1995) para el manganeso total en esta misma zona de estudio. La disminución del $\mathrm{pH}$ a bajas salinidades observada en este estudio, puede estar influyendo en la desorción del Mn del material en suspensión, ya que de acuerdo con la Fig. 3 , a valores de $\mathrm{pH}$ mayores a 8 unidades las concentraciones de Mn disminuyen. Wollast et al. (1979) señalaron que el manganeso se encuentra en forma de óxidos que experimentan reducción cuando disminuyen las concentraciones de oxígeno disuelto y decrece el $\mathrm{pH}$. BASKARAN \& SANTSCHI (1992) encontraron que la resuspensión de las partículas de sedimentos eran importantes en las concentraciones de este metal en la fracción particulada en la bahía de Galveston (Texas, EEUU), al igual que Morse et al. (1993) encontraron grandes cantidades de este metal que los vientos resuspenden en los sedimentos de esta misma bahía.

\section{Níquel}

La distribuciones del Ni contenido en el MES en relación con la salinidad se indican en la Fig. 5. Los aportes mensuales del Ni por el río, durante el estudio oscilaron entre no detectado y $0,250 \mu \mathrm{mol} / 1$ para la fracción $\mathrm{F} 1$, no detectado-0,567 $\mu \mathrm{mol} / 1$ para F2 y de no detectado-1,186 $\mu \mathrm{mol} / 1$ para $\mathrm{F} 3$, con las mayores concentraciones durante el período de lluvia. Igualmente, se observó remoción del metal durante la mezcla de las aguas, con aportes en

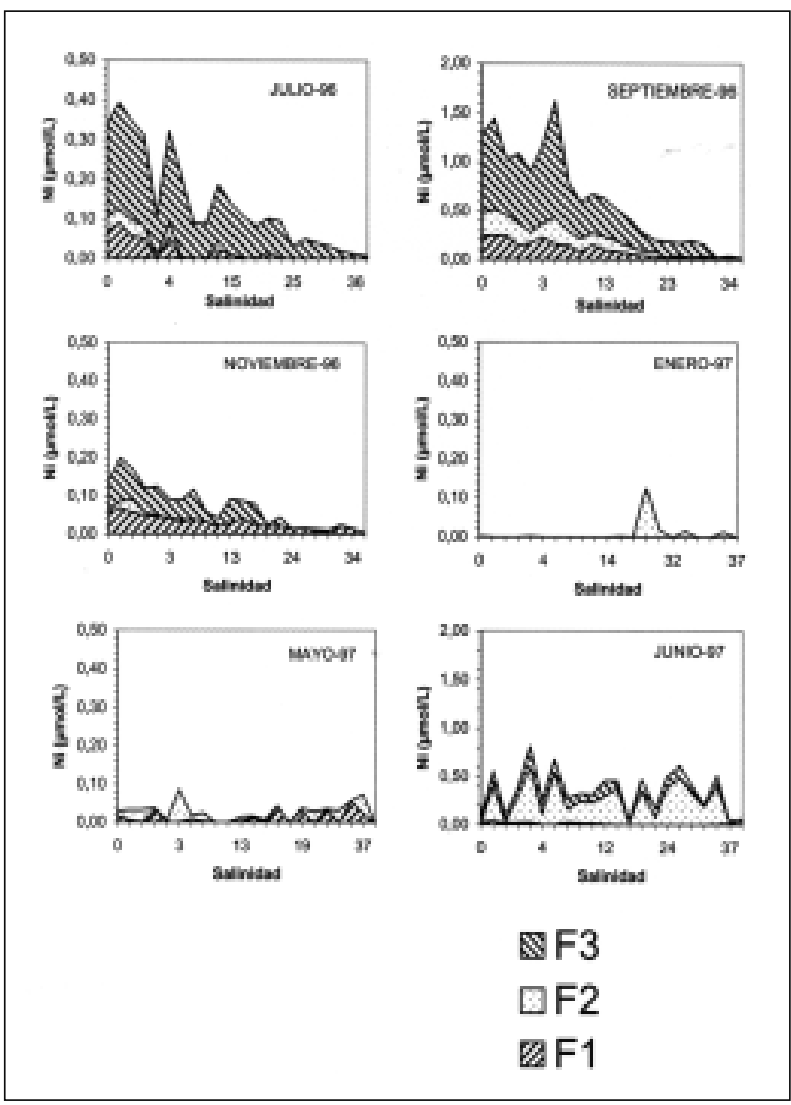

Fig. 5. Relación entre el Ni contenido en el MES y la salinidad en las aguas superficiales del río Manzanares y su pluma. La escala del eje y (conc. $\mu \mathrm{mol} / 1)$ cambia de magnitud en el mes de septiembre.

algunos puntos producto de los vertidos de efluentes líquidos y desechos sólidos a ambas márgenes del río y costa cercana a la desembocadura. La composición del MES, de igual manera, varió en cada uno de los muestreos, presentándose las tres fracciones durante la temporada lluviosa, mientras que durante la estación seca, la fracción perteneciente a oxihidróxidos de hierro y sulfuros metálicos es la única que se detectó en todos los meses a excepción de marzo que no se encontró Ni en el material en suspensión transportado por el río. Este comportamiento del $\mathrm{Ni}$ en el MES puede estar indicando que dicho metal está mayormente asociado con dicha fracción. Por otra parte, durante los meses de enero y mayo se observaron aportes de $\mathrm{Ni}$ asociados a los oxihidróxidos de hierro y sulfuros metálicos por encima de los 13,00 de salinidad, así como de Ni biodisponible en mayo, producto del dragado de la zona y de aportes provenientes de los astilleros, muelles y puertos establecidos cerca de la desembocadura. 
MÁrquez (1997) reportó remociones de Ni en el MES en esta misma área de estudio en un rango de 11-58 \% en el período de sequía y de 10-79\% en el de lluvia, mientras que LEÓN (1995) reportó remociones de Ni total en un rango de $25-86 \%$ y $50-86 \%$ en cada período. En este trabajo, las remociones estuvieron entre 0 y $55 \%$ durante todo el estudio. Las remociones pueden ser debidas a la coprecipitación del $\mathrm{Ni}$ con los oxihidróxidos de $\mathrm{Fe}$ y $\mathrm{Mn}$ coloidales que se forman durante la mezcla de las aguas. ZHANG (1995) señaló que el Ni manifiesta remoción desde el particulado, desorción desde la fase sólida en suspensión y desde los sedimentos del fondo, debido a los procesos geoquímicos. En este estudio, puede apreciarse la alta correlación del Ni con el MES durante el período de lluvia (Tabla II). Morse et al. (1993) también reportaron correlación del $\mathrm{Ni}$ particulado con los óxidos de hierro en la bahía de Galveston.

El comportamiento anormal del níquel es bastante complejo en muchos estuarios, debido a que participa en los procesos biogeoquímicos. En este estudio presentó un comportamiento no conservativo; sin embargo, Márquez (1997) reportó una aparente conservatividad en algunos meses para la pluma del río Manzanares, al igual que MARTIN et al. (1993) para el estuario del río Lena (Rusia).

El comportamiento no conservativo del $\mathrm{Ni}$ ha sido reportado en el estuario de Delaware (EEUU), debido a floculación geoquímica y a procesos de desorción desde el sedimento a salinidades entre 0 y 15 (SHARP et al. 1982; Apte et al. 1990). Zhang (1995) indicó que las concentraciones de $\mathrm{Ni}$ en el estuario Jiulungjiang eran más bajas en agua dulce que en la de mar y que la distribución presentó una desviación relativa de la línea de dilución teórica, hallándose decrecimiento en la concentración del metal en los sedimentos suspendidos en la zona de mezcla de las aguas dulces con las marinas, debido a los procesos de desorción de la superficie de las partículas de sedimentos. Estudios más recientes señalan que el Ni puede ser liberado desde los sedimentos a la columna de agua, teniendo asociaciones y perfiles semejantes a los nutrientes especialmente fosfatos y silicatos (YeAts et al., 1995). Así mismo, un comportamiento conservativo debido a la desorción desde la superficie de los sólidos ha sido señalado para el níquel por ELBAZPoulichet et al. (1996) en el estuario del río Rhöne (Francia).
Plomo

Los resultados para el plomo en el MES se muestran en la Fig. 6. Al igual que para los demás metales estudiados, el $\mathrm{Pb}$ particulado presentó un comportamiento no conservativo con las mayores concentraciones durante el período de lluvia con correlación altamente significativa entre la concentración total de $\mathrm{Pb}$ y el material en suspensión (Tabla II). En el mes de julio se presentaron las tres fracciones en el extremo fluvial (salinidades entre $0 \mathrm{y}$ $1,65)$, con aportes del metal que van desde no detectado en el puente Raúl Leoni hasta $0,052 \mu \mathrm{mol} / 1$ para el $\mathrm{Pb}$ biodisponible (F1), desde no detectado hasta 0,097 $\mu \mathrm{mol} /$ 1 para el $\mathrm{Pb}$ asociado a F2 y 0,030-0,042 para el $\mathrm{Pb}$ asociado a F3, el cual desapareció rápidamente del material en suspensión en la zona de mezcla y hacia la región marina. En septiembre la composición del $\mathrm{Pb}$ en el MES cambió, desapareciendo la fracción perteneciente al material refractario, evidenciando en este mes un comportamiento casi conservativo con aportes y remociones en la zona de mezcla, mientras que los niveles de plomo estuvieron en el rango de 0,011 a $0,082 \mu \mathrm{mol} / 1$ para la fracción F1 y entre 0,003 y $0,097 \mu \mathrm{mol} / 1$ para la F2. En noviembre no se presentaron aportes de $\mathrm{Pb}$ desde el río, observándose adición del metal en la F2 a todo lo largo de la región de mezcla de las aguas del río y de mar. Las posibles fuentes del plomo al sistema son los productos de la combustión de la gasolina debido al intenso tráfico de embarcaciones tipo peñero que constantemente transitan por la zona, la presencia de una estación de gasolina y diesel en la desembocadura del río, muelles pesqueros donde atracan los barcos atuneros, arrastreros y ferrys para el transporte de vehículos y personas a la Isla de Margarita y Península de Araya, así como de los colectores cloacales y canales de drenaje que vierten sus aguas en esta zona, llegando a la no detección del metal en la región netamente marina.

Durante el período de sequía, el $\mathrm{Pb}$ se presentó únicamente durante el mes de enero y en la fracción F1 con concentraciones entre 0,012 y $0,019 \mu \mathrm{mol} / 1$ con fuertes aportes en la zona de mezcla y a salinidades superiores a 30 , que pueden deberse al dragado de la zona que resuspende los sedimentos del fondo con la posible liberación del $\mathrm{Pb}$ adsorbido en la superficie de las partículas a la columna de agua. En marzo y mayo no se detectó Pb en ninguna de las fracciones separadas del material en suspensión. En junio, se presentaron aportes de $\mathrm{Pb}$ particulado biodisponible desde el río y de esta fracción y asociado a F2 en la zona de mezcla, el cual refleja el inicio 


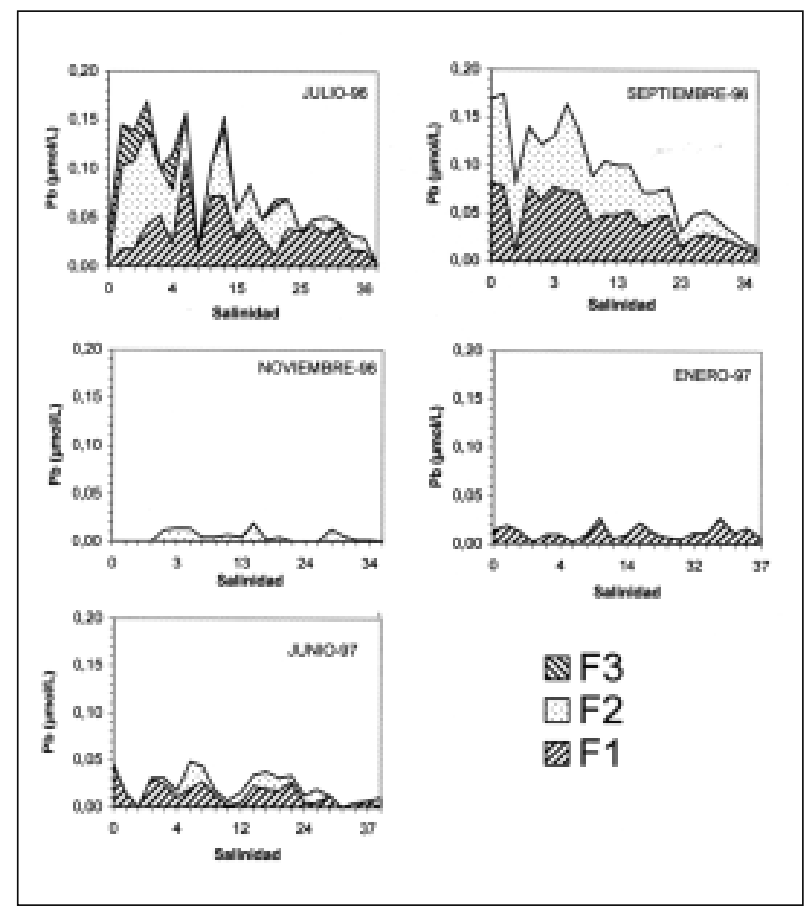

Fig. 6. Relación entre el Plomo en el MES y la salinidad en las aguas superficiales del río Manzanares y su pluma durante el presente estudio.

de la temporada de lluvia para el río de 1997, la cual produce el lavado de la corteza terrestre, vertiendo hacia el río y la costa todos los desechos acumulados en la superficie terrestre, lagunas y quebradas de la región.

El pH y la fuerza iónica del agua de mar pueden ser las causas principales de la incorporación del plomo en el material en suspensión transportado por el río. En efecto, a medida que aumenta el pH, disminuyen las concentraciones de plomo (Fig. 3). Otra posible causa de la adición del plomo al material en suspensión, es la participación de los oxihidróxidos de hierro y manganeso a través de sus coloides de carga negativa que se forman durante la mezcla del agua dulce y marinas. Según SADiQ (1991), el Pb suele ser adsorbido en la superficie de las partículas sólidas de Fe y Mn, las cuales, en la interfase agua de ríomar, tienden a precipitar debido a los cambios de $\mathrm{pH}$ y de la fuerza iónica. Esto parece ser confirmado por la correlaciones positivas muy significativas entre el $\mathrm{Pb}$ con el Fe y con el Mn durante los meses de julio y septiembre (Fig. 7 y Tabla II).

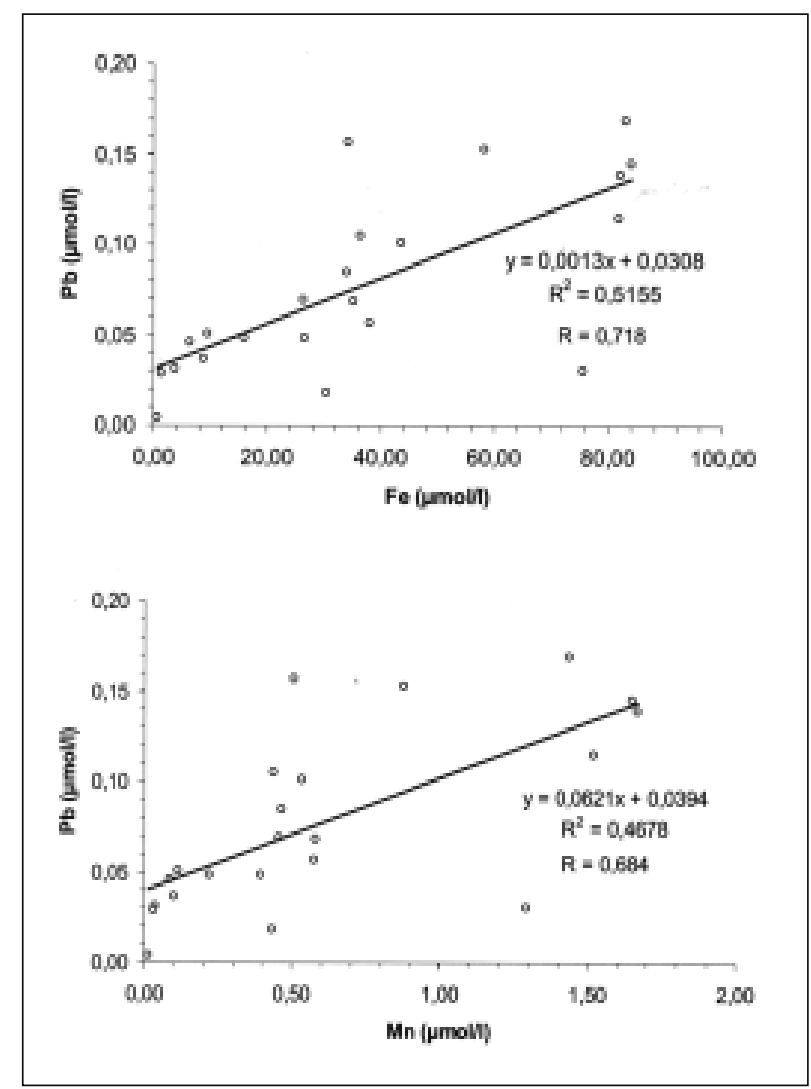

Fig. 7.- Relación entre el $\mathrm{Pb}$ y los metales $\mathrm{Fe}$ y $\mathrm{Mn}$ en las aguas superficiales de la cuenca baja y pluma del río Manzanares durante el mes de Julio de 1996.

Por otra parte, la presencia de plomo solamente en las fracciones biodisponible y potencialmente biodisponible (oxihidróxido de Fe y sulfuros metálicos), nos está indicando, que el contenido de $\mathrm{Pb}$ en los minerales que componen la corteza terrestre en la cuenca hidrográfica del río Manzanares es casi despreciable y el enriquecimiento de dicho metal en el material en suspensión, puede ser una consecuencia directa de todas las actividades antropogénicas que se realizan en la región y evidencia el impacto que producen los desechos de la ciudad de Cumaná en este ecosistema. RIEDEL \& SANDERS (1998) también observaron un enriquecimiento de $\mathrm{Pb}$ en el material en suspensión (seston) en el río Delaware y su estuario, estableciendo una escala de biodisponibilidad potencial $(\mathrm{Cd}>\mathrm{Zn}>\mathrm{Pb}>\mathrm{Cu}>\mathrm{Ni}>\mathrm{AS}>\mathrm{Cr}>\mathrm{Se})$. HART \& DAVIS (1981) señalaron que en el estuario del río Yarrah (Australia) una parte del $45 \%$ del plomo total es transportado en el MES. En este estudio, el $\mathrm{Pb}$ introducido al sistema estuarino del río Manzanares parece estar 
reflejando las observaciones hechas por estos autores, ya que este metal fue detectado en mayor proporción en forma particulada, presentando gran correlación con el MES (Tabla II). De igual manera, SALINAS et al. (1995) reportaron elevadas concentraciones de plomo debido a la corrosión de las tuberías de las aguas urbanas. Song \& Múller (1995) indicaron que gran cantidad del plomo encontrado en el río Neckar (Alemania) se debió a la descomposición de la biomasa, mientras que PEREIRA et al. (1995) señalaron que valores de 2,27 y 2,36 $\mu \mathrm{mol} / 1$ se hallaron en el agua del río Basin (Portugal), debido a la resuspensión de los sedimentos contaminados debido a los bajos valores de $\mathrm{pH}$.

Las estimaciones de los metales pesados Fe, Mn, Ni y $\mathrm{Pb}$ contenidos en el MES a partir de las correlaciones metal total vs MES, para el mes de septiembre se presentan en la Tabla III. Grandes cantidades de $\mathrm{Pb}$ y Ni están siendo transportadas desde el extremo superior de la cuenca baja hacia el mar, que pueden afectar la biota marina de toda la región costera aledaña a la ciudad de Cumaná. Por otra parte, las aguas de la cuenca baja del río son utilizadas para fines domésticos, agropecuarios e industriales. La población marginal ubicada en sus riberas utiliza sus aguas para el aseo personal y la preparación de alimentos, incorporando estos metales en su dieta con el correspondiente peligro para su salud.

Al comparar las concentraciones totales de los metales pesados determinados en el río Manzanares con los valores establecidos en las normativas del Ministerio del Ambiente y de los Recursos Naturales (M.A.R.N). para las aguas clasificadas como Tipo 4 (aguas destinadas a balnearios, deportes acuáticos, pesca deportiva, comercial y de subsistencia), en los subgrupos 4A (aguas para el contacto humano parcial) y 4B (aguas para el contacto humano total), los cuales indican que no deben detectarse metales pesados en estos cuerpos de agua, se encuentra que dicho río supera abiertamente estos valores.

Durante el período de lluvia, el aumento en las concentraciones del material en suspensión supone problemas de erosión de suelos debido a tala, quemas, prácticas agrícolas y pecuarias inadecuadas y a la extracción indiscriminada de arenas del lecho del río, que con la intensificación de las lluvias ocasiona el arrastre de una mayor carga sedimentaria, con la consiguiente afectación de otros sectores cercanos al ecosistema estudiado. Tal es el caso de blanqueamientos de corales en la región de bahía de Mochima del Parque Nacional
TABLA III. Contenido de metales pesados en el MES estimados a partir de las correlaciones metal total vs MES $(\mathrm{y}=\mathrm{mx}+\mathrm{b}$, donde $\mathrm{y}$ es la concentración del metal, $\mathrm{x}$ es la concentración del MES y b el corte con el eje y), para el mes de septiembre.

\begin{tabular}{cc}
\hline METAL & CONCENTRACIÓN (mg/kg) \\
\hline $\mathrm{Fe}$ & $29.042,0$ \\
$\mathrm{Mn}$ & 412,0 \\
$\mathrm{Ni}$ & 93,0 \\
$\mathrm{~Pb}$ & 207,0 \\
\hline
\end{tabular}

Mochima, posiblemente debido a la deposición del material en suspensión transportado por el río Manzanares y que puede llegar hasta esta región.

Es importante conocer el efecto de la dinámica del río Manzanares sobre el ecosistema marino costero, mediante el establecimiento de radiales direccionales de acuerdo al flujo laminar de la pluma del río. Se debe muestrear diferentes estratos para estudiar la hidrogeoquímica de la mezcla vertical, igual que conocer el alcance direccional de la cuña salina. Lo último debe hacerse lo antes posible debido a los problemas de blanqueamiento de corales en la región de la bahía de Mochima, los cuales pueden ser producto del aumento de las concentraciones del material en suspensión como consecuencia de la erosión por la deforestación de su cuenca.

\section{CONCLUSIONES}

Los flujos de metales pesados desde el río Manzanares hacia la región costera bajo su influencia se incrementan durante el período de lluvia, lo que indica que dichos elementos están principalmente asociados al material en suspensión y en consecuencia con el gasto del río.

El proceso de remoción que experimentaron los metales pesados a bajas salinidades es debido a la floculación (principalmente formación de oxihidróxidos de $\mathrm{Fe}$ y $\mathrm{Mn}$ ) y rápida precipitación como consecuencia de los cambios en el pH y de fuerza iónica, que tiene lugar durante la mezcla de los dos tipos de agua y que desestabiliza la solución de agua dulce.

El material en suspensión presenta una alta proporción de metales que pueden estar adsorbidos o como carbonatos y oxihidróxidos de manganeso muy reactivos $(\mathrm{Mn}$ y $\mathrm{Pb})$, 
los cuales pueden estar biodisponibles debido a que pueden ser liberados muy fácilmente de las partículas en suspensión por los organismos superiores y por las bacterias. Por otra parte, el Fe y Ni están mayormente asociados a los minerales de arcilla (aluminosilicatos), los cuales revelan un origen litogénico y en el caso del $\mathrm{Ni}$, es más difícil su incorporación en la cadena trófica del ecosistema.

Existe una influencia directa entre la pluviosidad y el gasto del río en los aportes de metales al ecosistema. Esto puede constatarse con las relaciones lineales significativas entre los metales con el material en suspensión.

La remoción del $\mathrm{Pb}$ en el material en suspensión presenta una relación lineal positiva muy signicativa con los metales $\mathrm{Fe}$ y $\mathrm{Mn}$, indicando una coprecipitación con los oxihidróxidos de Fe y Mn que se forman por floculación en los primeros momentos de la mezcla de las aguas, debido a los cambios de $\mathrm{pH}$ y fuerza iónica.

\section{AGRADECIMIENTO}

Al personal técnico del Departamento de Oceanografía del Instituto Oceanográfico de Venezuela por su valiosa contribución en la obtención de la data. Este trabajo fue financiado por el Programa de Estudio Ambiental del río Manzanares del Departamento de Oceanografía.

\section{REFERENCIAS}

Aguilera, L. \& J. Rojas. 1976. La ictiofauna del complejo hidrográfico del río Manzanares. Lagena 37-38: 23 35 .

Apte, S., M. Gadner, A. Gunn, J. Ravenscroft \& J. Vale. 1990. Trace metals in the Severn estuary: A reappraisal. Mar. Poll. Bull. 21 (8): 393-396.

Baskaran, M. \& P. SAntschi. 1992. The role of particles and colloids in transport of radionuclides in coastal environment of Texas. Mar. Chem. 26: 67-79.

Carpenter, J., W. Bradford \& V. Grand. 1975. Processes affecting the composition of estuarine waters $\left(\mathrm{H}_{2} \mathrm{CO}_{3}, \mathrm{Fe}, \mathrm{Mn}, \mathrm{Cu}, \mathrm{Ni}, \mathrm{Cr}\right.$ y $\left.\mathrm{Co}\right)$. En: Estuar. Res. Cronin. L. (Ed.), Vol. I. Academic. Press. New York,
USA. p. 188-214.

Coonley, L., E. BaKer \& H. Holland. 1971. Iron in the Mullica River and in Great Bay. New Jersey. Chem. Geol. 7: 51-63.

Elbaz-Poulichet, F., J. Garnier, D. M. Guan, J. M. Martin \& A. J. THOMAs. 1996. The conservative behaviour of trace metals $(\mathrm{Cd}, \mathrm{Cu}, \mathrm{Ni}$ and $\mathrm{Pb})$ and $\mathrm{As}$ in the surface plume of stratified estuaries: Example of the Rhône River (France). Estuar. Coast. Shelf Sci. 42: 289-310.

GIBBS, R. 1977. Transport phases of transition metals in the Amazon and Yukon rivers. Geol. Soc. Amer. Bul. 88 (5-8): 829-843.

Graham, W., M. Barder \& G. Klinkhammer. 1976. Manganese in Nagarranset Bay. Limnol. Oceanogr. 21 (1-6): 665-683.

HART, B. \& S. Davis. 1981. Trace metals speciation in the freshwater and estuarine regions of Yarrah River (Victoria). Estuar. Coast. Shelf. Sci. 12 (4): 353-374.

LANDING, W.M. \& B. L. LeWIS, 1991. Collection, processing and analysis of marine particulate and colloidal material for transition metals. In: Marine Particles: Analysis and characterization. D. C. Hurd \& D. W. Spencer (Eds.). American Geophysical Union. Washington D.C. USA. pp. 263-272.

León, I. 1995. Distribución y comportamiento de los metales pesados (Fe, $\mathrm{Mn}, \mathrm{Ni}, \mathrm{Cr}, \mathrm{Cu}, \mathrm{Cd}, \mathrm{Pb}$ y $\mathrm{Zn}$ ) en la cuenca baja y pluma del río Manzanares, Cumaná- Venezuela, durante el período comprendido entre Marzo y Noviembre de 1994. Trab. Grad. M. Sc. Ciencias Marinas, Univ. Oriente, Cumaná, Venezuela, 214 pp.

, W. Senior \& G. Martínez. 1997. Comportamiento el hierro, cromo, cadmio y plomo total en las aguas superficiales del río Manzanares. Venezuela, durante los períodos de sequia y lluvia en el año 1994. Caribb. J. Sci. 33 (1):105-107.

LewIS, B. L. \& W. M. LANDING. 1992. The investigation of dissolved and suspended-particulate trace metal fractionation in the Black Sea. Mar. Chem. 40: 105141. 
Magnusson, B. \& L. Rasmusseu. 1982. Trace metal levels in coastal sea water. Mar. Poll. Bull. 13 (3): 81-84.

Márquez, A. 1997. Comportamiento y distribución de algunos metales pesados en fracciones disueltas y particuladas en aguas superficiales del río Manzanares, Estado Sucre, Venezuela. Trabajo de grado Lic. en Química. Esc. de Ciencias. Univ. Oriente. $141 \mathrm{pp}$.

, W. Senior \& G. Martínez. 2000. Concentraciones y comportamiento de metales pesados en una zona estuarina de Venezuela. Interciencia 25 (6): 284291.

Martin, J. \& H. Windom. 1990. Present and future rol of ocean margin in regulation marine biochemical cycle of trace elements. Mar. Chem. 36: 15-67.

, D. Guan, F. Elbaz-Poulichet, A. Thomas \& V. Gordev. 1993. Preliminary assessment of the distribution of some trace elements $(\mathrm{As}, \mathrm{Cd}, \mathrm{Cu}$, $\mathrm{Fe}, \mathrm{Ni}, \mathrm{Pb}$ y $\mathrm{Zn}$ ) in a pristine aquatic environment in the Lena River estuary (Russia). Mar. Chem. 43: 185-199.

Martínez, G. \& W. Senior. 2001. Especiación de metales pesados $(\mathrm{Cd}, \mathrm{Zn}, \mathrm{Cu}$ y $\mathrm{Cr})$ en el material en suspensión de la pluma del Río Manzanares, Venezuela. Interciencia. 26 (2): 53-61.

Morris, A. \& A. BALE. 1979. Effect of rapid precipitation of dissolved $\mathrm{Mn}$ in river waters on estuarine $\mathrm{Mn}$ distribution. Nature 229: 303-327.

Morse, J., B. Presley, R. Taylor, G. Benoit \& P. Santschi. 1993. Trace metal chemistry of Galveston Bay: water, sediments and biota. Mar. Environ. Res. 36: $1-37$.

Palanques, A. \& J. Díaz. 1994. Anthropogenic heavy metal pollution in the sediments of the Barcelona continental shelf (Northwestern Mediterranean). Mar. Environ. Res. 38: 17-31.

Pereira, E., Y. Moura, J. Costa, J. Mahony \& R. Thomann. 1995. The Sto. Domingo mine: A study of heavy metals contamination in the water column and sediments of the Chanca River Basin by discharge from an ancient cupriferous pyrite mine (Portugal). Mar. Freshwater Res. 46: 145-151.

Riedel, G. \& J. SAnders. 1998. Trace element speciation and behavior in the tidal Delaware River. Estuaries 21 (1): 78-90.

Salinas, J., J. Ruiz \& G. Frances. 1996. Heavy metal levels in intertidal sediments and biota from Bidasoa estuary. Mar. Poll. Bull. 32 (1): 69-71.

SEnior. W., \& G. Godoy. 1990. Estudio físico-químico del río Manzanares, Cumaná- Venezuela. Bol. Inst. Oceanogr. Venezuela, Univ. Oriente 29: 160-172

. 1989. Estudio hidroquímico del estuario del río Manzanares: I Elementos Nutritivos . III Congreso Latinoamericano sobre Ciencias del Mar (COLACMAR). Cumaná-Venezuela.

Sharp, J., C. Culberson \& T. Church. 1982. The chemistry of the Delaware estuary. General considerations. Limnol. Oceanogr. 27 (6): 1015-1028.

SONG. Y. \& G. MülLER. 1995. Biogeochemical cycling of nutrients and trace metals in anoxic freshwater sediments of the Neckar River, Germany. Mar. Freshwater Res. 46: 237-243.

Vallette-SiLVER, N. 1993. The uses of sediment cores to reconstruct historical trends in contamination of estuarine and coastal sediments. Estuaries 16 (3b): 577-588.

Wollast, R., G. Billen \& J. Duinker. 1979. Behavior of manganese in the Rhine and Scheldt estuaries. Physics and chemical behavior. Estuar. Coast. Mar. Sci. 8: 161-169.

YeAts, P. 1993. Input of metals to the North Atlantic from two large Canadian estuaries. Mar. Chem. 43: 201209.

, \& S. Westerlunnd \& A. Flegal. 1995. Cadmium, copper and niquel distributions at four stations in the eastern central and south Atlantic. Mar. Chem. 42: 283-293.

ZHANG, J. 1995. Geochemistry of trace metals from Chinese 
River/Estuary Systems: An Overview. Estuar. Coast. Shelf Sci. 41: 631-658.

RecibIDO: Septiembre 2004

ACEPTADO: Agosto 2005
Zhang, J., W. Huang, S. Lin, M. Liu, Q. Yu \& H. Wang. 1992. Transport of particulate heavy metal towards the China Sea: A Preliminary study and comparison. Mar. Chem. 40 (3-4): 61-178. 